\title{
Coronavirus disease 2019: a revolution in biological triage in the emergency setting
}

\author{
Shahram Manoochehry*, Fatemeh Sabouri**, Mehrdad Faraji*, \\ and Mohammad Javad Behzadnia*@
}

\begin{abstract}
Triage is a familiar concept for all who work at the forefront of the treatment of patients and the emergency medical staff know their duty in different situations including crisis and epidemics. Accordingly, the coronavirus outbreak has also a major effect on the emergency department (ED) as it changes the routine ED function. Our main question was what are the main triage challenges in the recent pandemic and how we could overcome these challenges? Therefore, a search of the main Web databases was performed for articles published till May $1^{\text {st }}, 2020$ using various related keywords. In various steps, the title, summary, methodology, results, and discussion of the selected studies were assessed to find out the recent triage strategies in the COVID-19 outbreak. Hence, all the available and related English review articles, case series, and experimental studies were evaluated. Among 200 studies initially reviewed, 59 met the study criteria for the final assessment. COVID-19 puts a significant load on public health services and potential damage to social and psychiatric situations by marked morbidity and mortality. In line with the various presentations and according to the changing of the COVID-19 epidemic to a worldwide pandemic problem, the management and treatment protocols changed several times. Accordingly, the local and even global hospital protocols were changed as well. The first simple concept of coronavirus triage in an emergency department is the separation of COVID-19 infected patients from the others. This approach has been practiced around the world. Changing the ED layout from a usual triage or fast track set to an isolated room is necessary for such a pandemic situation. It is very important to consider staff communication and the application of PPE. All the efforts should be taken to protect patients as well as the medical staff from unnecessary exposure and infection; this serves to keep the health facilities working well in the outbreak and diffusion of SARS-CoV-2.
\end{abstract}

Keywords: COVID-19, SARS-CoV-2, triage, emergency medicine, coronavirus
*Trauma Research Center, Baqiyatallah University of Medical Sciences, Tehran, Iran **Department of Pathology, Shohada Hospital, Qom University of Medical Sciences, Qom, Iran

\section{Correspondence:}

${ }^{\circledR}$ Mohammad Javad Behzadnia, Trauma Research Center, Baqiyatallah University of Medical Sciences, Tehran, IR Iran, Nosrati Alley, Sheikh Bahaie St, Molla Sadra St, Vanaq Sq., Tehran, IR Iran Tel.: +98-2181262012, Email:Behzadnia@bmsu.ac.ir ORCID ID: 0000-0002-3062-0839

Date of first submission, October 4, 2020

Date of final revised submission, November 30, 2020

Date of acceptance, December 7, 2020

This open access article is distributed under a Creative Commons AttributionNon Commercial-Share Alike 4.0 International License

Cite this article as: Manoochehry S, Sabouri F, Faraji M, Behzadnia MJ. Coronavirus disease 2019: a revolution in biological triage in the emergency setting. Univ Med 2020;39:212-23. doi: 10.18051/UnivMed.2020.v39. 212-223 


\section{INTRODUCTION}

Triage is a familiar concept for all who work at the forefront of the treatment of patients; almost all of the emergency medical staff know their duty in different situations including crises and epidemics. Within the outbreak of Coronavirus 2019 and the changing into a pandemic, triage found a new meaning which will be discussed here. The coronavirus outbreak has also a major effect on the emergency department (ED) as it changes the routine ED function. Nowadays, triage becomes challenging in various situations during the current COVID-19 pandemic; as the clinician should decide which non-COVID infection patient should be hospitalized due to the other critical reasons, indeed, they should do this by risk assessment and outcome evaluation.

According to different policies, triaging occurs at the entrance into the emergency department and may differ in various hospitals. Three, four, or five-level triage systems are commonly used in various parts of the world; the latter seems to be more helpful under normal circumstances, but in this pandemic as a biological attack, it necessitates a change in the routine triage protocol. With the passing of the time and symptom variations, the emphasis on vital signs during triage also changed, given that the purpose of triage in biological crises is to achieve maximum benefits and mortality - morbidity reduction. The novel coronavirus (COVID-19) which now has spread worldwide was first distinguished in Wuhan, China in late December 2019 and was verified by the World Health Organization as a pandemic on March 1, 2020 while only 118000 cases were globally reported in the whole world. ${ }^{(1,2)}$

This pandemic at a large scale put a burden on the world's health infrastructures. In line with the various presentations and according to the changing of the COVID-19 epidemic to a worldwide pandemic problem, management and treatment protocols changed several times. Accordingly, the local and even global hospital protocols were changed as well.
Because this is a novel topic, to find previously published studies relevant to the current study question, a search of the Web of Science, Google Scholar, Cochrane, and PubMed databases was performed for articles published till May 1st, 2020 using the keywords "coronavirus", "corona", SARS-COV-2", “ SARS"," MERS" "Emergency Medicine", "emergency", "triage", "COVID-19", and "biology*". A combination search based on MESH terms was also performed. The PRISMA checklist was used to evaluate the validity of the selected articles. ${ }^{(3)}$ During the previous Middle East Respiratory Syndrome Coronavirus (MERS-COV) outbreak, Saudi Arabia used a visual triage scale for evaluating and scoring suspected cases; but further studies showed it had not enough effectiveness. ${ }^{(4)}$

In various steps, the title, summary, methodology, results, and discussion of the selected studies were assessed. Initially, the titles and abstracts were evaluated and saved to EndNote X8. Then, the full-text of English review articles, case series, and experimental studies were evaluated. Letters and duplicate studies were excluded. In the next stage, full texts were reviewed by two independent experts, and unrelated studies were excluded. Some similar and duplicate studies had no new information. Accordingly, some other papers for which the full text was not available were excluded in the second and third stages. Among 200 studies initially reviewed, 50 met the study criteria for the final assessment.

\section{Evidence-based triage changes}

COVID-19 puts a significant load on public health services and causes potential damage to social and psychiatric situations by its marked morbidity and mortality. In this condition, triage has found new meanings in various places such as surgical triage, psychological triage, etc. ${ }^{(5,6)}$

\section{Symptom-based triage}

The first simple concept of coronavirus triage in an emergency department is the 
separation of COVID-19 infected patients from the others. This approach has been practiced around the world since the early days of the pandemic but further evaluation showed no effectiveness as well.

In the early days of the problem, triage was mainly based on clinical symptoms such as fever, chills, and sore throat, along with controlling the percentage of blood oxygen saturation $\left(\mathrm{O}_{2} \mathrm{Sat} \%\right)$ and the other vital signs. In this protocol, $\mathrm{O}_{2} \mathrm{Sat} \%$ $(\leq 93 \%)$ and temperature $\left(\geq 37.8^{\circ} \mathrm{C}\right)$ were more emphasized and even became the criterion for further follow-up evaluations. ${ }^{(7)}$ In previous studies, $28.6 \%$ of confirmed coronavirus related respiratory cases had fever, but now, there are different reports from various countries (Table $1)$.

In a US healthcare system study; $30.7 \%$ of patients were febrile, $17.3 \%$ had a respiratory rate of more than 24 breaths/minute and $27.8 \%$ received supplemental oxygen during the triage. ${ }^{(8)}$ Smoking and co-infection with two or more viruses were the two significant predictors of fever.

Fever is less common among adults in viral infection than the children; in a study, more than $75 \%$ of adults with viral respiratory infection did not have a fever and this is what we see in the novel COVID-19 outbreak. During the first weeks, we have visited many febrile patients who had respiratory symptoms but when the outbreak spread globally most of the patients were afebrile. It seems that the virus symptoms are changing over time. ${ }^{(9)}$ Thus, the fever is not considered as an important criterion now.
Furthermore, there were some confirmed patients who had no symptoms at all. Field experiences over the past months have shown that many patients who come to the emergency room accidentally or for other reasons had COVID-19 positive tests, so there is a need for a modified evidence-based triage system at the early stages. Accordingly, even some routine triage criteria should temporarily change during the pandemic. More exact field investigations showed how medical staff and monitoring systems could change or correct the initial protocols through the current pandemic. According to recent experiences, some triage categories have now developed.

Consequently, some rare symptoms have been added to the above presentations and some cutaneous manifestations are reported as COVID-19 presentation and should be considered during the patient's triage. ${ }^{(10)}$ Neurological manifestations from mild to severe symptoms are also reported as the new COVID19 presentations. ${ }^{(11)}$

\section{Triage structure and design}

In the age of coronavirus-19, it is strongly advised to set up special hospitals, install quarantine wards, and establish preventive and controlled systems for infected patients. Furthermore, other hospitals could safely treat the other non-COVID-19 patients. Inpatient and outpatient units have to be separated in such a way that the patients who are in ED are not exposed to the risk of cross-infection at the early stages (Figure 1).

Table 1. The most common, less common and serious symptoms of COVID -19

\begin{tabular}{lll}
\hline Most common symptoms & Less common symptoms & Serious symptoms \\
\hline Fever & Aches and pains & $\begin{array}{l}\text { Difficulty breathing or shortness of } \\
\text { breath }\end{array}$ \\
Dry cough & Sore throat & Chest pain or pressure \\
Tiredness & Diarrhea & Loss of speech or movement \\
& Headache & \\
& Loss of taste or smell & \\
& A rash on skin, or discoloration of \\
& fingers or toes & \\
\hline
\end{tabular}




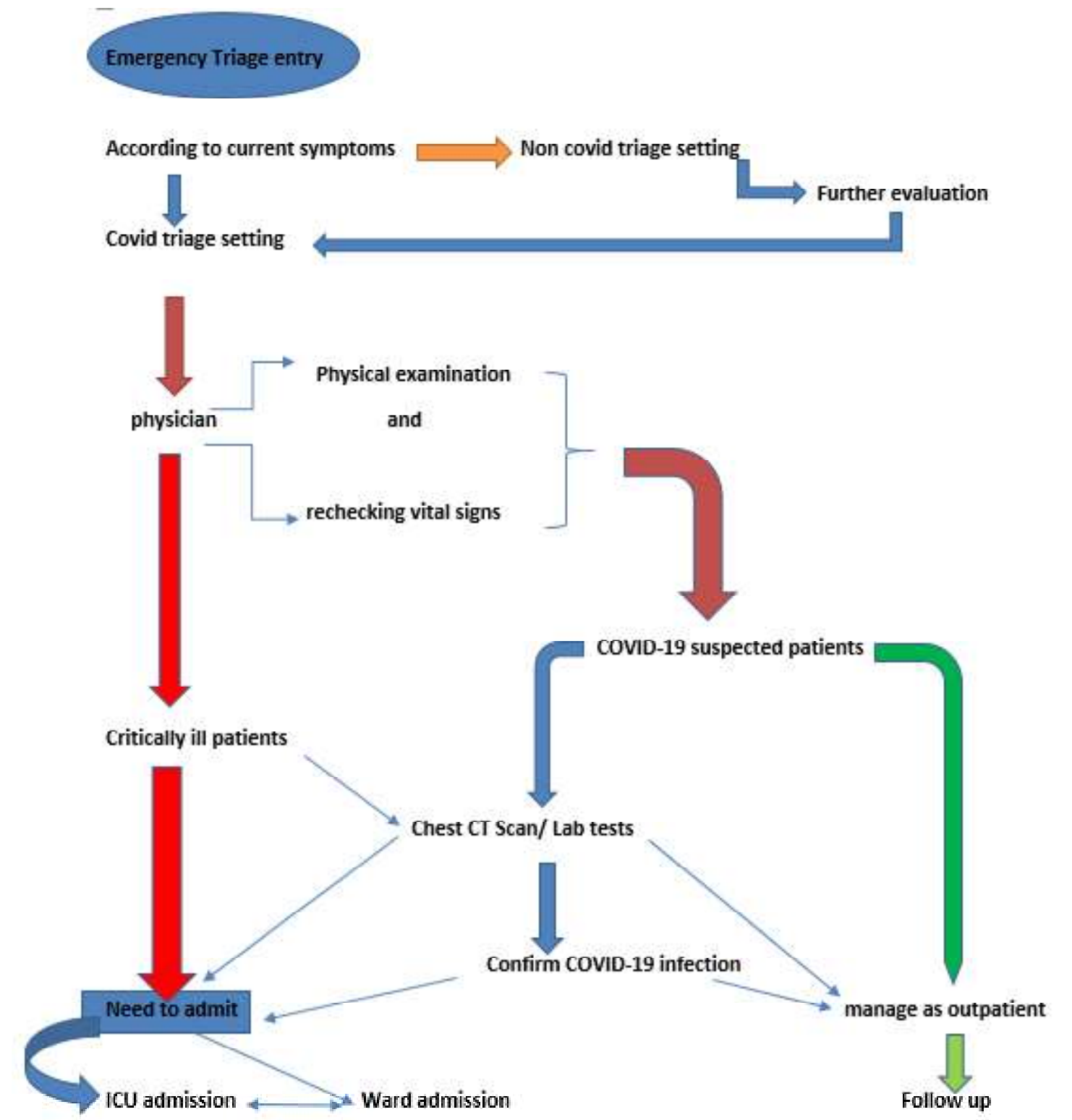

Figure 1. Emergency department triage in COVID-19 outbreak

In Italy, Emergency Departments (EDs) were reorganized to tackle the COVID-19 pandemic, including the introduction of pretriage and different care paths for patients. Structurally, the EDs were divided into separate compartments, termed clean and infected zones, respectively. According to pretriage results, regular non-COVID-19 were sent to the clean zones, whereas COVID-19 patients were sent to the infected zones. Both clean as well as infected patients subsequently underwent the standard triage procedure. ${ }^{(12)}$
Designing some field hospitals is necessary to avoid further referral hospital infection; Chinese experiences in developing Fangcang shelter hospitals are notable. These temporary hospitals have various essential functions such as isolation and triage, basic medical care, screening $_{2}$ and rapid referral parts for public health emergencies. ${ }^{(13)}$ Accordingly, appropriate designing for pre triage, diagnosis, and even isolation of doubtful or verified cases is also critical. Triage structure design in a hospital is now a multidisciplinary and collaborative task; 
as it has many different aspects, space definition, resource prioritization, supplies, infection control measures, staff selection, and many other factors need a widespread and multilevel protocol. It should also consider patient and medical staff safety. ${ }^{(14)}$

\section{TRIAGE SYSTEM CONSIDERATIONS BEFORE ADMISSION}

\section{Suspected coronavirus infection triage}

At the beginning of the pandemic in many parts of the world, workers in healthcare centers continued to visit coronavirus patients in addition to their routine tasks; but gradually the burden of the patients reached such a level that some additional special centers were needed to treat them. Thereafter, more challenging issues arose in the triage of the patients. In this way, the traditional triage system could no longer result in the recognition of suspected coronavirus cases. Accepting non-corona patients, how to visit and refer them to the other centers, and how to triage and deal with acute presentations of COVID-19 infection became the new challenges. ${ }^{(15)}$

\section{Triage of high-risk patients against high- risk symptoms}

Changing the specific guidelines has also led to a revolution in emergency triage; the handling of many cases that normally needed to be investigated and treated urgently have been altered to observe more in non-life-threatening circumstances to avoid infection. The first group contains patients with a history of immunodeficiency for any reason, whether pharmacological or due to an underlying disease; the second group contains the suspected patients with other acute manifestations. Besides the lifethreatening situation, we may encounter patients who have high-risk and emergency symptoms; they may need acute surgical, gynecological, or other emergency interventions. Furthermore, emergency trauma care is another aspect that needs timelines of interventional effectiveness in the outbreaks, hence elective surgeries and procedures should be postponed for later times..$^{(15,16)}$

\section{A multidisciplinary team approach for triage Team work and collaboration in triage}

During the triage and in the next stages, the number of organ failures, survival prediction, and performance score could help to prioritize the critically ill patients in some situations. However, the proper decision should be made by experienced special teams. Certainly, regional, national, and even international collaboration is needed to overcome this pandemic. ${ }^{(17)}$

Emergency radiology, as a relatively new subspecialty, plays a critical role in both the diagnosis and triage of acutely ill patients, and provides leadership to coordinate with the radiology department. Then they could deal better with novel highly infectious patients. ${ }^{(18)}$ Emergency and radiology department coordination is critical, as the suspected respiratory patients should be referred for a chest X-ray or CT scan evaluation without delay. In this condition, all the patients with respiratory symptoms are considered as probable cases of COVID-19 until proven otherwise.

Risk stratification of COVID 19 patients (in terms of clinical condition, patient health, hospital resource availability) and proper decision-making by multidisciplinary teams could decrease the faults and minimize the hospital stay. On the other hand, focusing on restricted protocols may lead to admission of more negative patients and increasing the risk of in-hospital infectious transmission. A high level of compliance could be achieved only by continuous observation, constant surveillance, and feedback reporting to the medical staff (Figure 2.).

Nowadays, different protocols and approaches are needed for different situations and epidemics. Accurate triage for surgical procedures is needed not only by telephone at home before hospitalization but also at the time of admission (telemonitoring surveillance). ${ }^{(19)}$ 

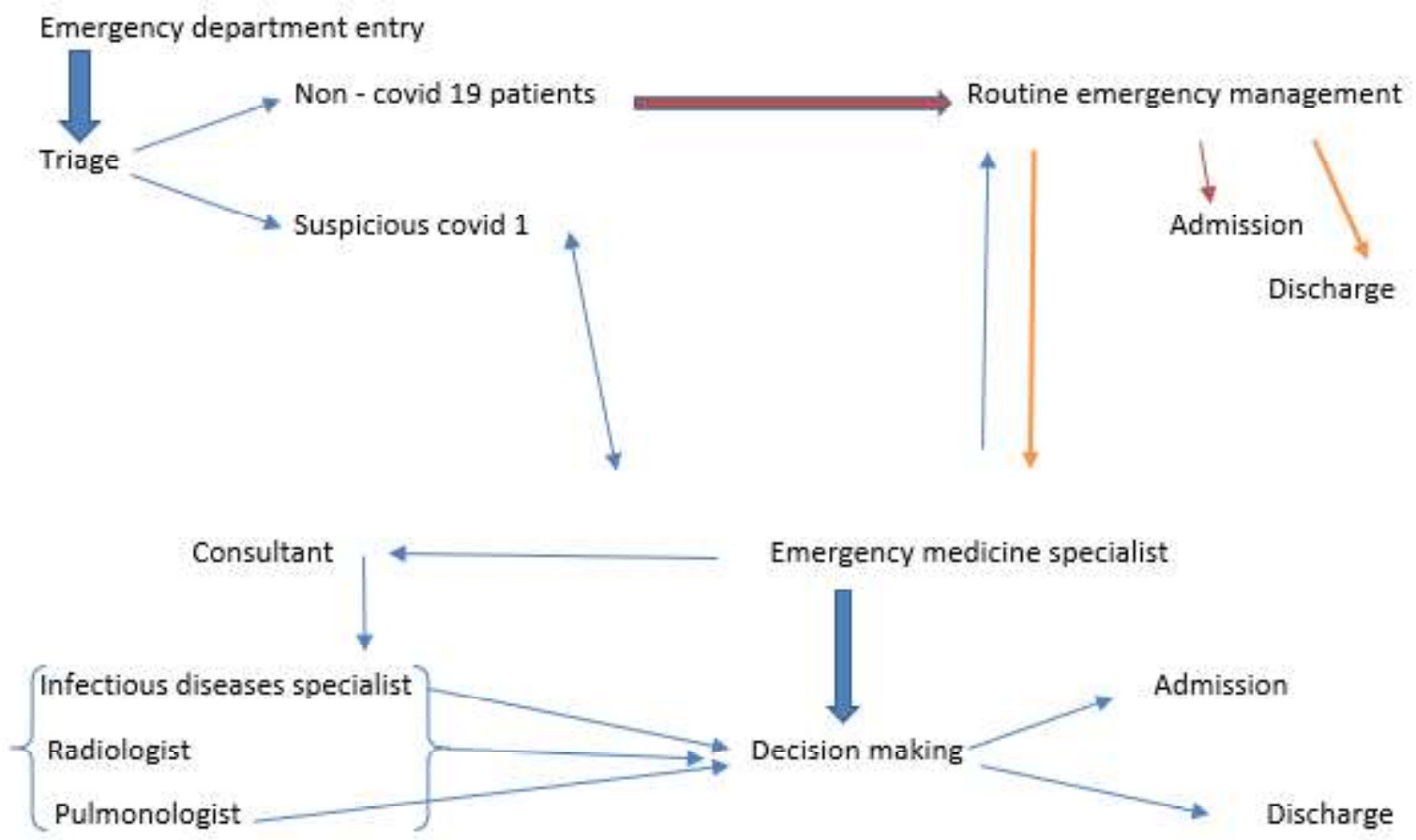

Figure 2. Team work and collaboration in triage

Surgeons need close collaboration with infection control specialists to avoid cross-infection in the hospital and to provide optimized treatment. Continued screening and triage have a primary preventive effect. ${ }^{(20)}$

\section{Special high-risk groups in triage}

The coronavirus outbreak requires special considerations on particular groups. As a highrisk population with more mortality and morbidities, the elderly with comorbidities require more special consideration. ${ }^{(21)}$ Subsequently, remote monitoring of infection risk in people who are on immunosuppressant drugs, during the COVID-19 pandemic is a possible and practical approach. Pregnant women are a susceptible high-risk group during this pandemic and therefore need special care and management in the emergency department. Anatomical feature and immunity alteration make the pregnant women susceptible to viral infections such as COVID-19; so all the maternal health settings should have a good management system to minimize their risk during the outbreak. ${ }^{(22)}$
Considering minimal contact could significantly decrease the risk. Prenatal care, proper triage, and recognition of high-risk cases, admission control, and more supervision are the main things that should be considered in maternal health care during the outbreak of COVID-19. ${ }^{(22)}$

COVID-19 has a significant effect on cardiovascular problems, such as increasing the risk of severity, complications, drug-related side effects, and death. Having an exaggerated search of COVID-19 related symptoms could affect non-coronavirus patients such as those with cardiovascular conditions. ${ }^{(23)}$ Any micro aerosol-generating procedure should be stopped during the COVID-19 pandemic. In previous outbreaks (2017-18), all age groups had the same mortality rate, but the males were interestingly more affected $(74.7 \%)$ with an equal mortality rate in both genders $(30.5 \%)$. It seems that the novel COVID-19 has a more significant morbidity rate than previous virus outbreaks. Therefore, current triage systems should be more aware of their hazardous and infective features. ${ }^{(24-26)}$ 


\section{SPECIAL CONSIDERATIONS FOR HEALTH CARE WORKERS}

\section{Personnel education and safety in triage}

Changing the ED layout from a usual triage or fast track set to an isolated room is necessary for such a pandemic situation. It is very important to consider staff communication and the application of personal protective equipment (PPE). All the efforts should be taken to pretest patients as well as the medical staff from unnecessary exposure and infection; this results in keeping the health facilities working well in the outbreak and diffusion of SARS-CoV-2. The importance of infection control is therefore crucial in limiting the effects of virus diffusion.

Proper and regular healthcare professions training and education are necessary during the COVID-19 outbreak, in which occupational and health safety are the two main components. ${ }^{(27)}$ Procedures that produce aerosols such as advanced airway management or cardiopulmonary resuscitation (CPR) have a significant risk. Hence, the emergency department personnel need to be well equipped with appropriate PPE and trained in their use. ${ }^{(28)}$

Guidelines of triage should contain a protocol for appropriate use of personal protective equipment and precautions to be taken in every stage of the management and help for allocating scarce resources. The reinforcement of specific infection control training is necessary for medical staff.

As in the previous viral infections, the novel coronavirus-19 clinical presentation varies from symptomatic cases to severe fatal disease. Healthcare involvement could easily occur among an overcrowded poor compliance infection control setting and unrecognized infected people could easily be missed in a poor triage system. ${ }^{(29)}$

Experiences from the outbreaks of the Middle East Respiratory Syndrome Coronavirus (MERS-COV) showed multiple healthcare association involvement. Some studies showed inappropriate donning and doffing of PPE and N95 mask fit testing among the healthcare workers who encountered the patients directly; so the application of special protective protocols could minimize the risk of cross-infection significantly. (30) As their direct contact with COVID-19 patients, healthcare providers are at high risk of infection transmission during the triage and treatment procedures; so having a contact distance of at least 2 meters and wearing a special mask and other PPE, could play a strong role.

\section{The role of laboratory data in triage}

In some medical centers, taking a chest $\mathrm{CT}$ scan is considered as a screening route for retriage and admission of non-corona patients who have other critical conditions in the ED. Problems and critical illnesses such as acute abdomen have a high incidence in the emergency room, requiring selective or emergency surgery.

Epidemiological and preliminary screening of viral nucleic acids; taking of chest CT examination during the perioperative period to rescreen COVID-19 and risk reduction of droplet and aerosol transmission, should be performed to reduce patient's susceptibility and shorten the length of postoperative hospital stay. In some conditions, the physician could triage the suspected febrile patients with simple laboratory data such as complete blood count (CBC) with a differential count. ${ }^{(31)}$ Some other modalities such as lung ultrasonography can also help to find the involved patients and facilitate the triage process. It could determine the workflow of the affected patients to the next step of care. ${ }^{(32)}$ The physician may visit some asymptomatic patients who have taken a laboratory test on their own initiative and are bringing the test results themselves. The diagnostic testing should be simplified and available to healthcare providers to allow them to decide more rapidly and accurately. ${ }^{(33)}$

This categorization could determine the severity and level of infection; subsequently, proper protective equipment is required. MERSCOV patients presented with monocytosis, normal white blood cell (WBC) count, and a lower C - reactive protein (CRP); but leukopenia and lymphopenia with mild eosinopenia and 
elevated CRP ( $\geq 4 \mathrm{mg} / \mathrm{L})$ are much more frequent in COVID-19 patients than in the controls. These laboratory findings could be helpful for better triage and treatment of febrile symptomatic patients in the ED. ${ }^{(31,33)}$ Evaluation of triage effectiveness is very important for hospital managers for future decision making. In this way, they could recognize the ultra-high risk, high risk, and moderate risk areas according to various risk stratifications. ${ }^{(33)}$

Proper clinical parameter definition for triage could help the staff to better manage the positive patients and lead to the best outcomes. Furthermore, the effectiveness of an emergency department in pandemic situations such as COVID-19 could be measured by a reduction in positive cases, admissions, and control of transinfectivity. So ED function could be improved by identification of the high-risk patients and proper admission. ${ }^{(34)}$ Establishing some isolation areas for triage is the first step to counter a pandemic surge such as COVID19. Exposure to unsuspected patients, lack of triage to use PPE and infection control, lack of awareness and preparedness place the health care providers at a high-risk level. ${ }^{(33,35,36)}$

\section{Tele triage and digital technology in COVID-19}

Methods of an effective and applicable triage setting are based on symptom severity and patient comorbidities. In addition, to confirm the virus transmission, all the involved health care systems should use information technologies. ${ }^{(37,38)}$ The role of telemedicine in resource conservation and remote patient care along with management strategies is obviously important. Whenever possible, medical staff should shift their visits away from the direct face to face visits to some other kinds such as telemedicine and video screening. ${ }^{(39)}$ Web-based monitoring and triage could somewhat help to overcome the limited equipment and prevent unnecessary healthcare exposures. ${ }^{(40)}$

Active triaging and screening for symptoms of COVID-19 infection could be considered for malignant and palliative care patients who are at their homes to avoid further infection dissemination. Telephonic screening could also help to assess their condition. ${ }^{(39,41)}$

Self-triage and self-scheduling plans are suggestive tools that the patients who are not critically ill could carry out themselves, explain their symptoms, and shorten the unnecessary triage times in the hospitals; thereby improving the triage efficacy and postponing all elective visits and surgical procedures. ${ }^{(42)}$ Accordingly; digital technologies could help the health organizations to have a valuable role in various stages of the COVID-19 outbreak, from screening and triage to diagnosis and monitoring. Video consultation in the triage of patients with COVID-19 is a way to increase the protection of personnel. ${ }^{(43,44)}$ Having a protective policy in triage is helpful for the allocation of the scarce resources when it is necessary to select the patients for urgent visits or telemedicine follow-up. ${ }^{(45)}$

Designing a digital triage tool to identify people with a high risk of COVID-19 infection is a possible approach for remote monitoring of highrisk patients, therefore, it could limit unnecessary access to healthcare or medical centers and reduce the spread of the infection. ${ }^{(46)}$ Delayed diagnosis, inappropriate use of PPE, and ED overcrowding are the main factors that are necessary for a triage setting.

The well-being of the health care providers is the first step of every medical care setting; hence all the policies should primarily focus on medical health care providers. Workload and psychiatric pressures could eventually lead to caregiver burnout; therefore, it affects medical care and patients' management. Using telemedicine and social media could partially prevent this workload. ${ }^{(47)}$

In a pandemic such as COVID-19, the emergency physicians as the frontline healthcare providers should be aware of ED triage, facilities, and isolation policies, to deal with a surge of suspected cases. The design of appropriate devices and procedures for pre-triage, diagnosis, and isolation of suspected and confirmed cases 
should be the final goal. ${ }^{(48)}$ Elective treatment and procedures should be postponed until the situation become more stable. ${ }^{(49)}$

The COVID-19 pandemic leads to delay of many routine treatments; hence it is necessary to have a risk assessment for the patients. Developing some new protocols for emergency management among the COVID-19 outbreak is essential. All specialties should have their own triage guidelines for their critical patients in the outbreaks. It is also necessary to update the previous protocols. Hence, they should design a conceptual framework for emergency and timely triage. ${ }^{(50,51)}$

In order to avoid loss of resources physicians with an overlap in therapies should have well-defined arrangements among each other concerning the treatment spectra.

Patient triage and risk assessment before any non-urgent procedures, resource allocation and prioritization, regular monitoring of personal protective equipment, infection control measures, and protective device training should be a part of every hospital against the COVID-19 infection.

Eventually, coronavirus outbreaks bring us some excellent lessons in the triage concept. First of all, as a biological threat, COVID-19 triage is not as simple as we think. In addition, coronavirus infection symptoms are more complex and various in different peoples. According to new conceptions, many medical policies are changed during these pandemics. Using some immune response assay, molecular and rapid $\mathrm{IgM} / \mathrm{IgG}$ testing could help to find the suspected patients more rapidly. Hence, early screening and triage may improve the response time in clinical and public health work, although their exact importance is still unknown. Considering the hospital status, ICU, and ventilator capacity or supplies limitations that are likely to evolve over the few hours or days, we saw a significant revolution in medical interrelationship, include some usual medical plans that were not more efficient and in need of modification. This biological threat also has many other lessons such as how to triage different patients with or without
COVID-19 symptoms and how to guide them to have laboratory and radiological assessment. It teaches our medical staff how to protect themselves against a non-visible threat in an emergency setting.

\section{CONCLUSION}

As mentioned, we may encounter some similar biological threats such as COVID-19 infections that even change the infrastructure parts of the medical systems. It seems that preparing the medical personnel and wellequipped hospitals is more serious in the future. furthermore, the bioethical points in various parts of triage and diagnosis to do proper treatment are crucial in patient management.

Finally, we should think about how to conserve hospital critical resources such as ICU beds, respirators, transmission capacity as well as protective gear (e.g. PPE for protection of the staff and patients against unnecessary exposure and intrahospital transmission. We hope this information could help the medical staff in all parts of the world.

\section{CONFLICT OF INTEREST}

None.

\section{ACKNOWLEDGEMENTS}

The authors would like to thank the "Clinical Research Development Unit of Baqiyatallah Hospital" for all their support, guidance and financial supports during carrying out this study.

\section{CONTRIBUTORS}

SM and MJB contributed to conception and design, MF helped in acquisition, analysis and interpretation of data. MJB and FS contributed to drafting the article, critical revision of the article and all of the authors contributed the final approval of the version to be published. 


\section{REFERENCES}

1. Hassan A, Arora RC, Adams C, et al. on behalf of the Canadian Society of Cardiac Surgeons, Cardiac surgery in Canada during the COVID-19 pandemic: a guidance statement from the Canadian Society of Cardiac Surgeons. Can J Cardiol 2020;36:952-5. DOI:https://doi.org/ 10.1016/j.cjca.2020.04.001.

2. Ross SW, Lauer CW, Miles WS, et al. Maximizing the calm before the storm: Tiered surgical response plan for novel coronavirus (COVID-19). JAm Coll Surg 2020;230:1080-91.e3. doi: 10.1016/ j.jamcollsurg.2020.03.019.

3. PRISMA (Preferred Reporting Items for Systematic Reviews and Meta-Analyses) Checklist. https://www.elsevier.com/_data/ promis misc/ISSM PRISMA Checklist

4. Alfaraj SH, Al-Tawfiq JA, Gautret P,Alenazi MG, Asiri AY, Memishet ZA. Evaluation of visual triage for screening of Middle East respiratory syndrome coronavirus patients. New Microbes New Infect 2018;26:49-52. doi: 10.1016/j.nmni. 2018.08.008.

5. Civantos FJ, Leibowitz JM, Arnold DJ, et al. Ethical surgical triage of head and neck cancer patients during the COVID-19 pandemic. Head Neck 2020;1-25.10.1002/hed.26229. doi: 10.1002/hed. 26229.

6. Valenza F, Papagni G, Marchianò A, et al. Response of a comprehensive cancer center to the COVID19 pandemic: the experience of the Fondazione IRCCS-Istituto Nazionale dei Tumori di Milano. Tumori J 2020:1-10. doi: 10.1177/ 0300891620923790.

7. Wang Q, Wang X, Lin H. The role of triage in the prevention and control of COVID-19. Infect Control Hosp Epidemiol 2020;1-5. doi: 10.1017/ ice.2020.185.

8. Richardson S, Hirsch JS, Narasimhan M, Crawford JM, McGinn T, Davidson KW, and the Northwell COVID-19 Research Consortium. Presenting characteristics, comorbidities, and outcomes among 5700 patients hospitalized with COVID-19 in the New York City area. JAMA 2020;323:20529. doi:10.1001/jama.2020.6775.

9. Korupolu R, Stampas A, Gibbons C, Jimenez IH, Skelton F, Verduzco-Gutierrezet M. COVID-19: screening and triage challenges in people with disability due to spinal cord injury. Spinal Cord Ser Cases 2020;6:35. https://doi.org/10.1038/ s41394-020-0284-7.

10. Wollina U, Karadað AS, Rowland-Payne C, Chiriac A, Lotti T. Cutaneous signs in COVID-19 patients: a review. Dermatol Ther 2020;e13549. doi: 10.1111/ dth. 13549 .

11. Werner C, Scullen T, Mathkour M, et al. Neurological impact of coronavirus disease (COVID-19): practical considerations for the neuroscience community. World Neurosurg 2020;139:344-54. doi: 10.1016/j.wneu.2020.04.222.

12. Turcato G, Zaboli A, Pfeifer N. The COVID-19 epidemic and reorganisation of triage, an observational study. Intern Emerg Med 2020;15: 1517-24. https://doi.org/10.1007/s11739-02002465-2.

13. Chen S, Zhang Z, Yang J, et al. Fangcang shelter hospitals: a novel concept for responding to public health emergencies. Lancet 2020;395:1305-14. DOI: https://doi.org/10.1016/S0140-6736(20)30744 -3 .

14. Carenzo L, Costantini E, Greco M, et al. Hospital surge capacity in a tertiary emergency referral centre during the COVID-19 outbreak in Italy. Anaesthesia 2020;75:928-34. doi:10.1111/anae. 15072.

15. Li Y, Zeng L, Li Z, et al. Emergency trauma care during the outbreak of corona virus disease 2019 (COVID-19) in China. World J Emerg Surg 2020;15: 33. https://doi.org/10.1186/s13017-020-00312-5.

16. Zimmermann M, Nkenke E. Approaches to the management of patients in oral and maxillofacial surgery during COVID-19 pandemic. J Craniomaxillofac Surg 2020;48:521-6. https:// doi.org/10.1186/s13017-020-00312-5.

17. Sprung CL, Joynt GM, Christian MD, Truog RD, Rello J, Nates JL. Adult ICU triage during the coronavirus disease 2019 pandemic: who will live and who will die? Recommendations to improve survival. Crit Care Med 2020;48:1196-202. doi: 10.1097/CCM.0000000000004410.

18. Nasir MU, Roberts J, Muller NL, et al. The role of emergency radiology in COVID-19: from preparedness to diagnosis. Can Assoc Radiol J 2020; 71:293-300. DOI: 10.1177/0846537120916419.

19. Simonato A, Giannarini G, Abrate A, et al. Clinical pathways for urology patients during the COVID19 pandemic. Minerva Urol Nefrol 2020;72:37683. doi: 10.23736/S0393-2249.20.03861-8.

20. Zou J, Yu H, Song D, Niu J, Yang H. Advice on standardized diagnosis and treatment for spinal diseases during the coronavirus disease 2019 pandemic. Asian Spine J 2020;14:258-63. DOI: 10.31616/asj.2020.0122.

21. de Marinis F, Attili I, Morgantiet S, et al. Results of multilevel containment measures to better protect lung cancer patients from COVID-19: the IEO model. Front Oncol 2020;10:665. doi: 10.3389/ fonc. 2020.00665 . 
22. Chen Y, Li Z, Zhang YY, Zhao WH, Yu ZY. Maternal health care management during the outbreak of coronavirus disease 2019. J Med Virol 2020;92:731-9. doi: 10.1002/jmv.25787.

23. Driggin E, Madhavan MV, Bikdeli B, et al. Cardiovascular considerations for patients, health care workers, and health systems during the COVID-19 pandemic. J Am Coll Cardiol 2020;75:2352-71. DOI: 10.1016/j.jacc.2020.03.031.

24. Mobaraki K, Ahmadzadeh J. Current epidemiological status of Middle East respiratory syndrome coronavirus in the world from 1.1.2017 to 17.1.2018: a cross-sectional study. BMC Infect Dis 2019;19:351. https://doi.org/10.1186/s12879019-3987-2.

25. Tan KA, Thadani VN, Chan D, Oh JYL, Liu GKP. Addressing coronavirus disease 2019 in spine surgery: a rapid national consensus using the Delphi method via teleconference. Asian Spine J 2020;14:373-81. https://doi.org/10.31616/asj.2020. 0224.

26. Lai THT, Tang EWH, Chau SKY, Fung KSC, Li KKW. Stepping up infection control measures in ophthalmology during the novel coronavirus outbreak: an experience from Hong Kong. Graefes Arch Clin Exp Ophthalmol 2020;258:1049-55. doi: 10.1007/s00417-020-04641-8.

27. Modi PD, Nair G, Uppe A, et al. COVID-19 awareness among healthcare students and professionals in Mumbai Metropolitan Region: a questionnaire-based survey. Cureus 2020;12: e7514. doi: 10.7759/cureus.7514.

28. Maudet L, Sarasin F, Dami F, Carron PN, Pasquier M. Emergency medical services: COVID-19 crisis. Rev Med Suisse 2020;16(No 691-2):810-4.

29. Balkhy HH, Perl TM, Arabi YM. Preventing health care-associated transmission of the Middle East Respiratory Syndrome (MERS): our Achilles heel. J Infect Public Health 2016;9:208-12. https:// doi.org/10.1016/j.jiph.2016.04.006.

30. Al-Tawfiq JA, Rothwell S, Mcgregor HA, Khouri ZA. A multi-faceted approach of a nursing led education in response to MERS-CoV infection. J Infect Public Health 2018;11:260-4. doi: 10.1016/ j.jiph.2017.08.006.

31. Park GE, Kang CI, Koet JH, et al. Differential cell count and CRP level in blood as predictors for Middle East respiratory syndrome coronavirus infection in acute febrile patients during nosocomial outbreak. J Korean Med Sci 2017;32: 151-4. doi: 10.3346/jkms.2017.32.1.151.

32. Dudea SM. Ultrasonography and SARS-CoV 2 infection: a review of what we know and do not yet know. Med Ultrason 2020;22:129-32. doi: 10.11152/mu-2612.
33. Li Q, Ding X, Xia G, et al. Eosinopenia and elevated C-reactive protein facilitate triage of COVID-19 patients in fever clinic: a retrospective casecontrol study. EClinicalMedicine 2020;23: 100375. DOI:https://doi.org/10.1016/j.eclinm.2020.100375.

34. Varughese S, Read JGb, Al-khal AL, Saleh SA, El Deeb Y, Camerona PA. Effectiveness of the Middle East respiratory syndrome-coronavirus protocol in enhancing the function of an emergency department in Qatar. Eur J Emerg Med 2015;22:31620. doi: 10.1097/MEJ.0000000000000285.

35. Her M. How Is COVID-19 affecting South Korea? What is our current strategy? Disaster Med Public Health Prep 2020:1-3. doi: 10.1017/dmp.2020.69.

36. Tran DL, Lai SR, Salah RY, et al. Rapid deescalation and triaging patients in communitybased palliative care. J Pain Symptom Manage 2020;60:e45-47. doi: 10.1016/j.jpainsymman.2020. 03.040 .

37. Krausz M, Westenberg JN, Vigo D, Spence RT, Ramsey D. Emergency response to COVID-19 in Canada: platform development and implementation for eHealth in crisis management. JMIR Public Health Surveill 2020;6:e18995. doi: 10.2196/18995.

38. Montesi G, Biase SD, Chierchini S, et al. Radiotherapy during COVID-19 pandemic. How to create a no fly zone: a Northern Italy experience. Radiol Med 2020:1-4. doi: 10.1007/s1 1547-02001217-8.

39. Judson TJ, Odisho AY, Neinstein AB, et al. Rapid design and implementation of an integrated patient self-triage and self-scheduling tool for COVID-19. J Am Med InformAssoc 2020;27:8606. doi: 10.1093/jamia/ocaa051.

40. Alwashmi MF. The use of digital health in the detection and management of COVID-19. Int J Environ Res Public Health 2020;17:2906. https:// doi.org/10.3390/ijerph17082906.

41. John O. Video consultations for triage of patients with covid-19. BMJ 2020;369:m1583. doi: https:// doi.org/10.1136/bmj.m1583.

42. Indini $\mathrm{A}$, Aschele $\mathrm{C}$, Cavanna $\mathrm{L}$, et al. Reorganisation of medical oncology departments during the novel coronavirus disease-19 pandemic: a nationwide Italian survey. Eur $\mathrm{J}$ Cancer 2020;132:17-23. doi: 10.1016/j.ejca.2020. 03.024.

43. Bonavita S, Tedeschi G, Atreja A, Lavorgna L. Digital triage for people with multiple sclerosis in the age of COVID-19 pandemic. Neurol Sci 2020;41:1007-9. doi: 10.1007/s10072-020-04391-9.

44. Moazzami B, Khorasani NR, Moghadam AD, Farokhi E, Rezaei N. COVID-19 and telemedicine: Immediate action required for maintaining 
healthcare providers well-being. J Clin Virol 2020; 126:104345. doi: 10.1016/j.jcv.2020.104345.

45. Costantini M, Sleeman KE, Peruselli C, Higginson IJ. Response and role of palliative care during the COVID-19 pandemic: a national telephone survey of hospices in Italy. Palliat Med 2020;34:889-95. doi: 10.1177/0269216320920780.

46. Etkind SN, Bone AE, Lovell N, et al. The role and response of palliative care anh Hospice services in epidemics and pandemics: a rapid review to inform practice during the COVID-19 pandemic. J Pain Symptom Manage 2020;60:e31-40. doi: 10.1016/j.jpainsymman.2020.03.029.

47. Taheri MS, Falahati F, Alireza Radpour A, et al. Role of social media and telemedicine in diagnosis \& management of COVID-19: an experience of the Iranian Society of Radiology. Arch Iran Med 2020;23:285-86. doi: 10.34172/aim.2020.15.

48. Moshayedi P, Ryan TE, Mejia LLP, Nour M, Liebeskind DS. Triage of acute ischemic stroke in confirmed COVID-19: large vessel occlusion associated with coronavirus infection. Front Neurol 2020;11:353. doi: 10.3389/fneur.2020.00353.
49. Oh MD, Choe PG, Oh HS, et al. Middle East respiratory syndrome coronavirus superspreading event involving 81 persons, Korea 2015. J Korean Med Sci 2015;30:1701-5. doi: 10.3346/jkms.2015.30.11.1701.

50. Agalar C, Engin DO, Protective measures for COVID-19 for healthcare providers and laboratory personnel. Turk J Med Sci 2020;50(Si-1):578-84. doi: 10.3906/sag-2004-132.

51. Pulia MS, O'Brien TP, Hou PC, Schuman A, Sambursky R. Multi-tiered screening and diagnosis strategy for COVID-19: a model for sustainable testing capacity in response to pandemic. Ann Med 2020;52:207-14. doi: 10.1080/ 07853890.2020 .1763449 . 